\section{The dangers of widespread nitric oxide screening for primary ciliary dyskinesia}

\section{ABSTRACT}

Primary ciliary dyskinesia (PCD) is underdiagnosed and requires complex testing at specialist diagnostic centres. Measurement of nasal nitric oxide (nNO) has good sensitivity and specificity screening for PCD, but is currently usually measured at PCD centres rather than prior to referral. Proposals to include NO testing for asthma diagnoses could widen access to PCD screening if nasal mode analysers are available. Data from 282 consecutive referrals to our PCD diagnostic centre (31 PCD positive) were used to model predictive values for $\mathrm{nNO}$ testing with varying pretest probability and showed that predictive values were good in the referral population, but extending screening to more general populations would result in excessive false positives that may overwhelm diagnostic services. Although nNO remains a useful test, a 'normal' result with classical clinical history should still be considered for further testing.

Proposed asthma diagnosis guidelines from the UK National Institute for Health and Care Excellence include the expansion of exhaled nitric oxide (NO) from a research tool into routine care. ${ }^{1}$ This could lead to improved availability of nasal NO (nNO) screening for primary ciliary dyskinesia (PCD).

Exceptionally low nNO differentiates PCD from other respiratory diseases ${ }^{2}$ and measurement is recommended as a screening test in symptomatic patients prior to diagnostic testing by high-speed video analysis (HSVA) of ciliary beating, transmission electron microscopy (TEM) of cilial ultrastructure or genetic tests. ${ }^{3}$ HSVA and TEM require expensive infrastructure and dedicated scientists; testing is focused in a geographically disparate centre making access for patients difficult. Local screening by measurement of $\mathrm{nNO}$ is therefore attractive; however, our data reiterate the importance of continuing rigorous patient selection in applying screening tests (pretest probability). Classical features suggestive of PCD include persistent wet cough, upper airway disease, neonatal respiratory distress and persistent rhinitis. ${ }^{4}$

We analysed nNO levels in 282 consecutive eligible referrals (aged 6-79 years) at our national PCD diagnostic centre (June 2006-December 2013) (see online supplementary methods for details of nNO measurement and PCD diagnosis). ${ }^{4}$ Thirty-one

Table 1 Sensitivity, specificity, PPV and NPV for different screening thresholds used across published studies on nNO diagnostics, ${ }^{2}$ but analysed against our data $(n=282)$

\begin{tabular}{|c|c|c|c|c|}
\hline & $120 \mathrm{~nL} / \mathrm{min}(\%)$ & $77 \mathrm{~nL} / \mathrm{min}(\%)$ & $40 \mathrm{~nL} / \mathrm{min}(\%)$ & $20 \mathrm{~nL} / \mathrm{min}(\%)$ \\
\hline Sensitivity & 100 (88.8 to 100$)$ & 93.6 (78.6 to 99.2$)$ & 93.6 (78.6 to 99.2$)$ & 77.4 (58.9 to 90.4$)$ \\
\hline Specificity & 68.9 (61.1 to 73.1$)$ & 84.1 (78.9 to 88.4$)$ & 94.4 (90.8 to 96.9$)$ & 97.6 (94.9 to 99.1$)$ \\
\hline PPV & 27.4 (19.5 to 36.6$)$ & 42 (30.2 to 54.5$)$ & 67.4 (51.5 to 80.9 ) & 80.0 (61.4 to 92.3$)$ \\
\hline NPV & $100(97.8$ to 100$)$ & 99.1 (96.7 to 99.9$)$ & 99.2 (97.0 to 99.9 ) & 97.2 (94.4 to 98.9 ) \\
\hline
\end{tabular}

out of 282 were PCD positive (11\%). A previously published threshold of $77 \mathrm{~nL} /$ $\min ^{5}$ was used; a level below this was considered a 'positive test'. Sensitivity in our population was $93.6 \%$ (95\% CI $78.5 \%$ to $99.0 \%)$ and specificity was $84.1 \%(95 \%$ CI $78.9 \%$ to $88.4 \%$ ) (table 1). Therefore, in our population the positive predictive value (PPV) was $42.6 \%$ (95\% CI 30.2\% to $54.5 \%)$ and negative predictive value (NPV) was $99.1 \%$ (96.6\% to $99.9 \%)$; so approximately $6 / 10$ patients with a positive test did not have PCD. However, predictive values were dependent on the prevalence of the disease in the tested population (figure 1). Previous data from children have suggested that the prevalence of PCD within those with recurrent wet cough is $5 \%,{ }^{6}$ which would give a PPV of $23.6 \%$ and NPV of $99.6 \%$; therefore, this group should be targeted for testing. A recent systematic review of 989 patients with non-cystic fibrosis bronchiectasis found that 9\% had PCD, which is a similar proportion to those referred to our service. ${ }^{7}$ Therefore, this is a population that could possibly be targeted for screening as long as other clinical features are present. PCD prevalence is around $1: 10000$ in the general population, ${ }^{8}$ which would reduce the PPV to less than $0.1 \%$, rendering general population screening useless. Additionally, nonspecialist centres need to understand how to interpret results; different cut-off values have been used that will result in varying accuracy metrics (table 1). Fractional exhaled NO is also lower in PCD than healthy/disease controls; however, it is less accurate than $\mathrm{nNO}^{9}$ and its use is not recommended by current PCD diagnostic guidelines. $^{10}$

These results are significant for several reasons. First, a NPV of $99.1 \%$ confirms that $\mathrm{nNO}>77 \mathrm{~nL} / \mathrm{min}$ is excellent for excluding PCD, although cases of PCD with normal nNO are well documented. ${ }^{2}$ Second, although a PPV of $42 \%$ makes $\mathrm{nNO}$ a poor diagnostic test in isolation, use prior to referral could reduce referrals and patient burden/healthcare costs associated with travel to a distant centre for complex diagnostics; indeed, 211 of our 282 patients would have had PCD correctly 'excluded' by nNO screening. Patients with a particularly strong history would warrant formal testing even with $\mathrm{nNO}>77 \mathrm{~nL} / \mathrm{min}$. Importantly, if $\mathrm{nNO}$ is used as a screening test in populations without classical symptoms of PCD, the number of false-positive cases will overwhelm diagnostic services.

PCD is underdiagnosed but increasing awareness and structured diagnostic

Figure 1 Relationship between PPV and NPV and the pretest probability of PCD (proportion of patients with PCD in the tested population). Sensitivity and specificity shown to illustrate that these are test specific and do not vary with disease prevalence. NPV, negative predictive value; PCD, primary ciliary dyskinesia; PPV, positive predictive value.

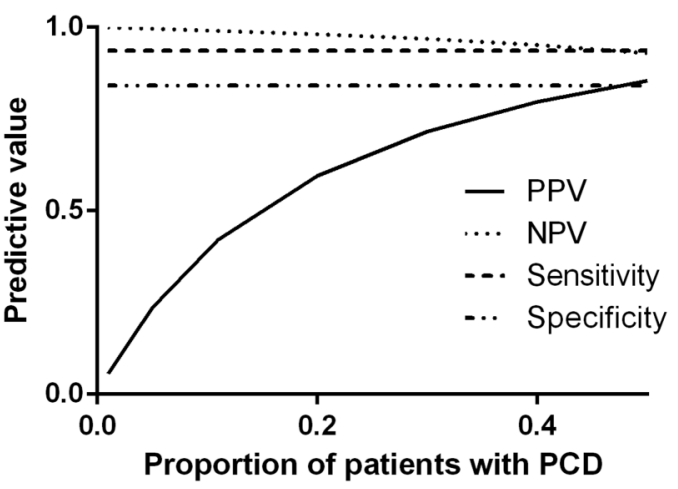


referral pathways are helping tackle this. ${ }^{3}$ Wider nNO use would rely on screening only those with classical symptoms of PCD and adequate competency-based training of those taking/interpreting $\mathrm{nNO}$ readings. Our measurements used a 'gold standard' breath-hold method with chemiluminescence analyser. Alternative breathing manoeuvres reduce discriminatory value $^{2}$ and portable machines without a 'real-time' nNO trace risk not taking plateau nNO readings. As $\mathrm{NO}$ analysers become more widespread, the opportunity for nNO screening at centres without PCD diagnostics is potentially useful if used in correctly selected patients.

\section{Samuel A Collins, ${ }^{1,2,3}$ Laura Behan, ${ }^{1,2,3}$ Amanda Harris, ${ }^{1,3}$ Kerry Gove, ${ }^{2,3}$ \\ Jane $S$ Lucas ${ }^{1,2,3}$}

${ }^{1}$ Primary Ciliary Dyskinesia Centre, University Hospital Southampton NHS Foundation Trust, Southampton, UK ${ }^{2}$ Clinical and Experimental Sciences Academic Unit, University of Southampton Faculty of Medicine, Southampton, UK

${ }^{3}$ NIHR Southampton Respiratory Biomedical Research Unit, University of Southampton and University Hospital Southampton NHS Foundation Trust, Southampton, UK

Correspondence to Dr Samuel Collins, Faculty of Medicine, MP810, LF100, Southampton General Hospital, Tremona Road, Southampton SO16 6YD, UK; samuel.collins@soton.ac.uk
Twitter Follow Samuel Collins at @samacollins

Contributors $\mathrm{AH}$ and $\mathrm{KG}$ conducted patient testing, LB collated and analysed data, SAC analysed data and drafted the manuscript with JSL.

Competing interests None declared.

Ethics approval Southampton and South West Hampshire Research Ethics.

Provenance and peer review Not commissioned; externally peer reviewed.

- Additional material is published online only. To view please visit the journal online (http://dx.doi.org/10. 1136/thoraxjn-2015-208056).

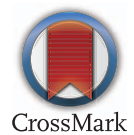

To cite Collins SA, Behan L, Harris A, et al. Thorax 2016;71:560-561.

Received 11 November 2015

Revised 14 January 2016

Accepted 25 January 2016

Published Online First 19 February 2016

Thorax 2016;71:560-561.

doi:10.1136/thoraxjnl-2015-208056

\section{REFERENCES}

1 Excellence $\mathrm{NI}$ for $\mathrm{H}$ and $\mathrm{C}$. Asthma: diagnosis and monitoring of asthma in adults, children and young people. 2015. https://www.nice.org.uk/guidance/ dg12/resources/asthma-diagnosis-and-monitoringdraft-nice-guideline2 (accessed 25 Feb2015).
2 Collins SA, Gove K, Walker W, et al. Nasal nitric oxide screening for primary ciliary dyskinesia: systematic review and meta-analysis. Eur Respir $J$ 2014:44:1589-99.

3 Lucas JS, Burgess A, Mitchison HM, et al. Diagnosis and management of primary ciliary dyskinesia. Arch Dis Child 2014;99:850-6.

4 Jackson CL, Behan L, Collins SA, et al. Accuracy of diagnostic testing in primary ciliary dyskinesia. Eur Respir J 2016:47:837-48.

5 Leigh MW, Hazucha MJ, Chawla KK, et al. Standardizing nasal nitric oxide measurement as a test for primary ciliary dyskinesia. Ann Am Thorac Soc 2013;10:574-81.

6 Chapelin C, Coste A, Reinert P, et al. Incidence of primary ciliary dyskinesia in children with recurrent respiratory diseases. Ann Otol Rhinol Laryngol 1997;106:854-8.

7 Brower KS, Del Vecchio MT, Aronoff SC. The etiologies of non-CF bronchiectasis in childhood: a systematic review of 989 subjects. BMC Pediatr 2014;14:4.

8 Lucas J, Walker W, Kuehni C, et al. Primary ciliary dyskinesia. Eur Respir Mon 2011;54:201-7.

9 Walker WT, Liew A, Harris A, et al. Upper and lower airway nitric oxide levels in primary ciliary dyskinesia, cystic fibrosis and asthma. Respir Med 2013;107:380-6.

10 Barbato A, Frischer T, Kuehni CE, et al. Primary ciliary dyskinesia: a consensus statement on diagnostic and treatment approaches in children. Eur Respir J 2009;34:1264-76. 